\title{
Handling Rapid Interference Detection of Progressive Meshes Using Active Bounding Trees
}

\author{
Peiran Liu and Nicolas D. Georganas \\ University of Ottawa \\ Gerhard Roth \\ National Research Council of Canada
}

\begin{abstract}
We introduce a novel approach, multiresolution collision detection, for fast and exact interference detection on continuous level-of-detail (LOD) representations of arbitrary triangle meshes undergoing rigid-body motion. A new algorithm, active bounding tree (AB-Tree), is presented to accelerate interference queries of three-dimensional models, which dynamically change LODs at runtime. The idea behind the approach is to selectively adjust the LODs of objects in a virtual environment to maintain an overall low complexity of collision detection (CD), therefore guaranteeing an interactive frame rate. On one hand the utilization of computational resources is maximized; on the other hand a consistent and realistic virtual environment is presented to the user. We also introduce a LOD selection method for CD. Our implementation of the CD algorithm demonstrates significant performance improvement over existing algorithms for static LOD meshes. The complexity of the algorithm is analyzed and proved. Measurements are taken to compare with the analytical results of three sample models and strong agreements are achieved.
\end{abstract}




\section{Introduction}

The increasing expectation for immersive and interactive entertainment in computer graphics applications requires fast high-fidelity physics simulation [Hecker 00]. Collision detection (CD), as the core of physics simulation, is often a bottleneck in interactive applications such as video games. Typically, low detailed meshes are used for real-time collision detection. They are decoupled from high detailed meshes used for visual rendering, which leads to inconsistency in physics simulation. The running time of existing CD algorithms depends on the complexity of input models and the output collision configuration. Therefore, setting the resolution of the meshes for collision detection in building a virtual environment becomes an engineering trade-off between speed and accuracy. Those algorithms cannot handle the situation when the resolutions of the meshes are changed at runtime due to mesh refinement.

In this paper, we present a new CD approach which takes advantage of multiresolution technique to dynamically adjust the complexity of $\mathrm{CD}$ at runtime. For each geometric model, the level of simplification depends on the availability of the computational resources at instance such as CPU and memory, and

how realistic or consistent a simulation the user expects on the object in the virtual world. Thus desired performance is achieved.

\section{Background}

The primary focus of this section is to summarize the collision detection methods and the multiresolution techniques, and set the context for readers who are interested in implementing the presented CD algorithm.

\subsection{Collision Detection}

Many important CD methods have been developed for rigid models. Recent surveys of these methods can be found in [Jiménez et al. 01, Lin and Gottschalk 98]. Algorithms with the current best runtime for convex polytope collision queries take linear time. If the objects are not moving swiftly, the best runtime can be roughly constant.

The algorithms for collision queries between general nonconvex polygonal models are dominated by the BVH strategy. The time cost is mainly determined by the complexity of input model, the choice of bounding volume (BV), and the contact configuration of two models. Sphere-trees [Hubbard 96, Bradshaw and O'Sullivan 04], oriented bounding box (OBB) trees [Gottschalk et al. 96], axis-aligned bounding box (AABB) trees [van den Bergen 97], discrete orientation polytope (k-dop) trees [Klosowski et al. 98], swept sphere 
volume (SSV) trees [Larsen et al. 99], and CLOD [Otaduy and Lin 03] are examples.

In time-critical collision detection, the BVH provides higher precision as the collision query search moves down the hierarchy and allows output precision to be adapted to the computing time available [Hubbard 96]. However, spatial inaccuracy is inherent due to the lack of exact collision queries on primitives. This is especially important when contact normal and contact points are required to compute a plausible collision response.

\subsection{Mesh Simplification and Multiresolution Data Structures}

Previous mesh simplification works fall into two categories: multiple static LODs and single LOD with multiresolution. The idea of the multiresolution mesh or continuous LOD [Gross et al. 96, Taubin and Rossignac 98, Hoppe 96] is to create a data structure that can be employed to dynamically produce a mesh with any desired resolutions lying between the highest and the lowest number of polygons from the original mesh. Recent works have been focused on view-dependent simplification, which take into account viewing parameters in mesh simplification to speed up graphics rendering further [Luebke and Erikson 97, Hoppe 97, Xia et al. 97]. A general reference to LODs and mesh simplifications can be found in [Luebke et al. 02].

Many works for general polygonal models are related to progressive meshes (PM). PM is proposed by Hoppe for accelerating graphics rendering [Hoppe 96]. A triangle mesh can be represented at multiple LODs by performing a series of refinement operations. He has also developed a view-dependent simplification algorithm for progressive meshes [Hoppe 97]. Xia et al. [Xia et al. 97] have introduced a merge tree, which is built upon progressive meshes to enable real-time view-dependent rendering. Luebke and Erikson [Luebke and Erikson 97] define a tight octree called vertex tree, which enables hierarchical view-dependent simplifications. Taubin et al. [Taubin and Rossignac 98] demonstrate a surface partition scheme for progressive encoding of surface.

By applying multiresolution meshes to collision detection, the accuracy of $\mathrm{CD}$ can be adjusted at runtime and the collision queries can be potentially accelerated. However, very few algorithms have been proposed to handle the multiresolution collision detection. One possible solution would be to apply CD to static LODs. However, auxiliary data structures, high computational cost, and the substantial amount of required storage are some of the limitations of this solution. El-Sana and Varshney [18] proposed a multiresolution hierarchy for haptic collision detection. Otaduy and Lin [10] proposed a "dual hierarchy" that serves both as a BVH for accelerating collision queries and a multiresolution representation of the original model for computing contact information. However, they do not apply to massive and large-scale virtual 
environments. The $\mathrm{CD}$ approach presented in this paper is under the context of large-scale virtual environment. Therefore, our focus is to globally refine a large number of relatively less complex models in the virtual environment, rather than view-dependently refine a few very large and complex models with millions of polygons, such as a terrain model.

\section{Collision Detection Algorithm and Data Structure}

In this paper, we present a time-critical collision detection approach for multiresolution models. It is based on a new algorithm, active bounding tree or $A B$-Tree. We demonstrate this approach by applying the $\mathrm{CD}$ algorithm to $\mathrm{PM}$, the most often used format for efficient representation of a mesh in a progressive multiresolution data structure. The AB-Tree, based on AABB BVH, serves not only as a BVH for rapid interference detection between meshes at instant resolutions but also as a tool for tracking the current active faces in the original mesh and efficiently refitting the BVH structure. SOLID is a programming library for collision detection between polygonal models using AABB BVH [van den Bergen 02]. Another CD library, RAPID, can be used as a starting point to implement our approach on $\mathrm{OBB}$, a more tight-fitting BV [Gottschalk et al. 97]. We assume the collision detections are performed on triangle meshes. The generation of multiresolution three-dimensional models takes advantage of the PM and quadric error metrics [Garland and Heckbert 97]. The PM has advantages over some other multiresolution representations. It supports progressive transmission, progressive refinement, viewdependent refinement, surface crease preserving, and crack preventing. The quadric error metrics is a method to efficiently generate multiresolution representations of triangle meshes in an arbitrary nonmanifold topology. We use the QSlim 2.0 mesh simplification package to create PM representations [Garland 04]. Some original models used in the experiments (bunny, cow, bones, etc.) are collected from [Garland 04].

The multiresolution $\mathrm{CD}$ algorithm is composed of two parts: mesh refinement and collision queries. The mesh refinement is operated on a vertex hierarchy data structure. In this section, we focus on the collision queries and introduce the AB-Tree data structure and the BVH algorithm. To illustrate the AB-Tree, we present it under the PM context as an example. We therefore start with a brief introduction to the PM. It should be noted that the new approach presented in this paper could be extended to accommodate other multiresolution representations.

\subsection{Progressive Mesh and Mesh Refinement}

A PM is constructed by iteratively performing edge collapses on an arbitrary mesh $\hat{M}$. A simplified mesh (or a base mesh $M_{0}$ ) is stored along with the 


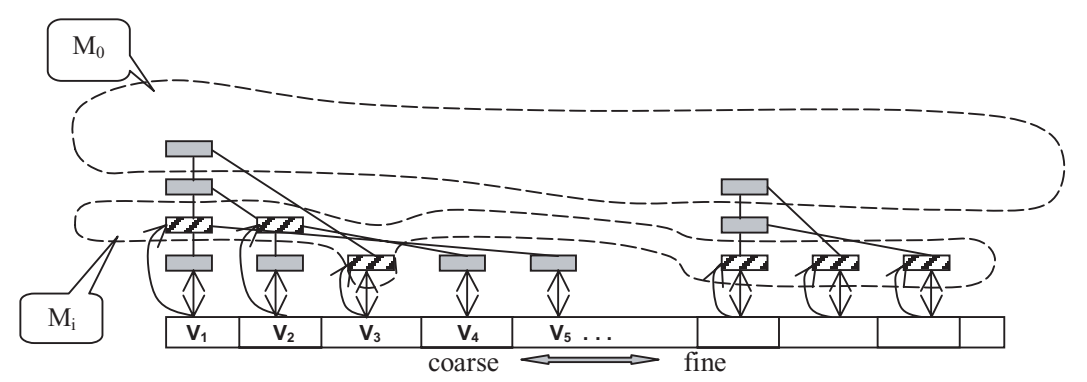

Figure 1. Illustration of a vertex forest.

inverse of all performed edge collapses (vertex splits) in a sequence of vertex split records vsplit $t_{i}$. Thus, a PM is generally represented as $\left(M_{0},\left\{v_{s p l i t}, \ldots\right.\right.$, vsplit $\left._{n-1}\right\}$ ). At runtime the PM is loaded into the vertex hierarchy, which is a multiresolution data structure similar to the "vertex tree" proposed by Luebke and Erikson in [14]. The vertex tree is a tight octree used in the dynamic mesh simplification algorithm for accelerating graphics rendering. Each tree node maintains one or more vertices. When the screen-space projection of a cell in the tree is too small, all the vertices in that cell are collapsed to one vertex. The polygons for rendering are maintained in an "active list." "Active tree" is a subtree of a vertex tree. It maintains a set of tree nodes whose vertices are already split. Vertex split operations are performed on the leaves of the active tree. The vertex hierarchy is composed of a vertex forest (a set of binary trees plus a vertex list) as shown in Figure 1 and a primitive list. Each tree node contains a vertex split record. We borrowed the idea of "active list" and use a vertex list to track the already split tree nodes. Any required mesh resolution can be obtained by performing a sequence of vertex splits or edge collapses on the vertex forest from the leftmost leaf node to the rightmost leaf node or vise versa. The data structure definition of the vertex hierarchy can be found online at the website listed at the end of this paper.

\subsection{Active Bounding Tree}

An AB-Tree is a BVH building upon a multiresolution mesh model. The ABTree data structure plus a series of operations upon it makes our CD algorithm support fast collision queries on multiresolution meshes, which are dynamically refined at runtime. Previously proposed BVH algorithms assume that mesh geometry and topology do not change at runtime. Our CD algorithm, however, breaks this constraint by efficiently refitting the BVH when the geometry and topology of the underlying triangle mesh are changed. Therefore, 
the $\mathrm{CD}$ algorithm can provide necessary performance. A detailed description of the AB-Tree data structure and the pseudocode of the operations are available online at the website listed at the end of this paper.

\subsubsection{Data Structure}

The AABB tree is introduced in [van den Bergen 97]. It provides a fast way to perform exact collision detection between complex models. Basically, it is a binary tree structure. A node $x$ in the tree has four fields: an AABB which bounds all AABBs of the leaves in the subtree rooted at $x$, range $[x]$; a link to the left child of $x$, left $[x]$; a link to the right child of $x$, right $[x]$; and a label to indicate the type of the node (leaf or internal), label $[x]$. The AABB of an internal node bounds the AABBs of its children nodes. The AABB of a leaf node bounds a geometric primitive. We use triangle face as the primitive to demonstrate our approach. The following property allows the cost of refitting an $\mathrm{AABB}$ in an $\mathrm{AABB}$ tree to be independent of the number of nodes in the tree.

Property Let $S$ be a set of primitives. $S+$ and $S-$ are subsets of $S . S+\subseteq S$, $S-\subseteq S, S+\cup S-=S$. If $B+$ and $B-$ are the smallest AABBs of $S+$ and $S-$, respectively, and $B$ is the smallest AABB enclosing $B+$ and $B-$, then $B$ is the smallest AABB of $S$ [van den Bergen 97].

Assume that range $[x]$ is defined as $\left[t_{1}, t_{2}\right]$, with low $(x)=t_{1}$ (the low endpoint) and $\operatorname{high}(x)=t_{2}$ (the high endpoint). Then following the above mentioned property, we have

$$
\operatorname{range}[x]=\left[\begin{array}{l}
\min (\operatorname{low}(\text { left }[x]), \text { low }(\operatorname{right}[x])), \\
\max (\text { high }(\text { left }[x]), \text { high }(\operatorname{right}[x]))
\end{array}\right] .
$$

An AB-Tree $T$ augments an AABB tree with additional information stored in tree nodes and an indexed list as illustrated in Figure 2. Besides the four

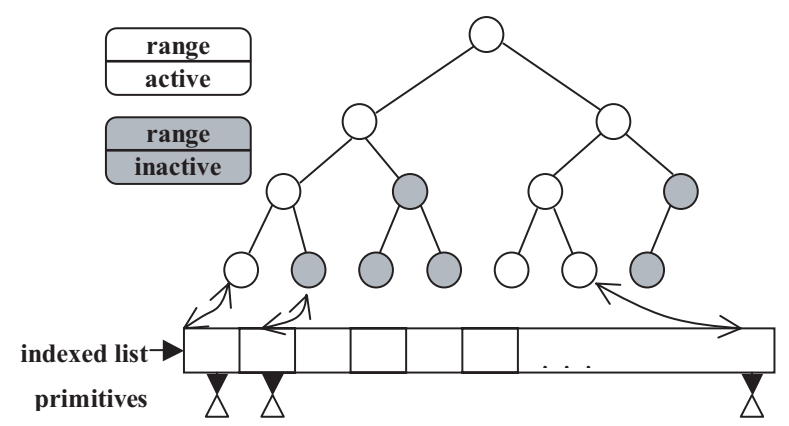

Figure 2. Illustration of the AB-Tree data structure. 
fields defined for the AABB tree, range $[x]$, left $[x]$, right $[x]$, and label $[x]$, every node $x$ in $T$ has three additional fields, a link to an element of the indexed list, index $[x]$; a link to the parent of $x$, parent $[x]$; and the status of the node $x$, status $[x]$, where status $[x] \in\{$ active, inactive, deformed $\}$.

An element $f$ in the indexed list has two fields, a link to a leaf node in the tree, leaf $[f]$; and a link to a primitive in the primitive list of the vertex hierarchy, primitive $[f]$. Every leaf in the AB-Tree is pointed from exactly one element of the indexed list.

CD algorithms supporting multiple resolutions require fast locating a leaf and all of its ancestors in a BVH given that the primitive bounded by the leaf is known. In an AABB tree, this may require searching several paths topdown due to the possible overlaps between the bounding boxes of the sibling nodes. However, in an AB-Tree, the index of the primitive can be used as a key to the indexed list so that a leaf, which bounds the primitive, can be located instantly. The parent field can then be used to find all the ancestors of the leaf in a bottom-up tracing. Compared with the AABB tree, it is clear that the AB-Tree has better performance. The purpose of the status field is discussed in the next section.

\subsubsection{Operations}

One of the key features of the PM is that only a few primitives of the original mesh are affected in each refinement. This means only a small number of bounding boxes in a BVH need to be refitted for collision detection. To quickly locate these bounding boxes, a bottom-up approach can be much faster than a top-down approach given that the affected primitives are known from the PM refinement as discussed in the last paragraph. The indexed list and the parent pointer in the AB-Tree are designed for this purpose. Once a node is located, the status field is used to identify those bounding boxes to be refitted. The status field has three possible values: deformed means that the bounding box needs to be refitted; inactive means that the bounding box is temporarily removed; active means that there is no change. Initially all nodes in the AB-Tree are set to active. All those deformed nodes form a small subtree as we can see in the following paragraphs. When a top-down traverse is performed on the subtree, the refitting is applied to each deformed bounding box. Comparing with the AABB tree, which requires a traverse on the whole tree, our AB-Tree algorithm is much faster. An AB-Tree maintains a set of dynamically changed bounding boxes of a BVH. Before we describe the detailed operations, we need to introduce some notations.

$P$ is a PM. $T$ and $T^{\prime}$ are two AB-Trees. $x$ is a node in $T . F$ is the indexed list of $T . T_{s}$ is a subtree of $T . I, R$, and $D$ are three sets of indices of the primitives in $P . \quad R \cap D=\varnothing . \quad I \cap D=\varnothing . \quad I \cap R=\varnothing$. At the current resolution of $P, I$ contains the indices of the primitives to be inserted into the 
mesh, $R$ contains the indices of the primitives to be removed from the mesh, $D$ contains the indices of primitives to be deformed in the mesh.

An AB-Tree supports the following operations.

1. ACTIVE-BUILD $(P)$ returns an AB-Tree $T$. An AB-Tree is constructed top-down, by recursive subdivision. The operation first defines all primitives stored in $P$ as a set. The smallest bounding box of the set is computed. The set is then split by ordering the primitives with respect to a well-chosen partitioning plane. The smallest bounding boxes for the two subsets are computed and the two subsets are then further partitioned. This recursive process terminates when each subset contains one element. The partitioning plane is chosen orthogonal to the longest axis of the bounding box and intersecting at a partitioning coordinate on the axis. The heuristic used in choosing a partitioning coordinate is subdividing the set of primitives into two sets of equal size. Thus, the AB-Tree $T$ becomes an AVL tree. If the original set has $n$ primitives, the AB-Tree $T$ has $n$ leaves. Another heuristic proposed in [van den Bergen 97] is to choose the median of a bounding box as the partitioning coordinate. However, when the geometric primitives are not distributed uniformly within their bounding box, this approach may construct an unbalanced tree. We show in the following section that an unbalanced AB-Tree does not perform very well in a BVH refitting process. The field leaf of every element in the list $F$ is set to link to a leaf in the tree $T$, which bounds the primitive. The index field of the leaf is set to link to the corresponding element in $F$.

2. ACTIVE-MARK $(I, R, D, T)$ marks the AB-Tree $T$ bottom-up and returns a subtree $T_{s}$ as shown in Figure 3(a). The logic behind this

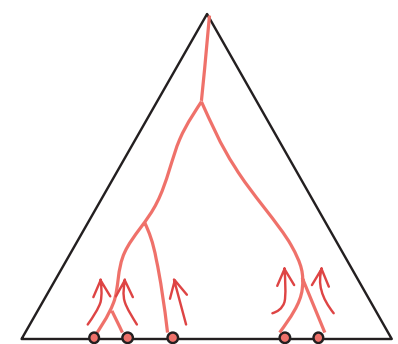

(a) Marking a tree bottom-up

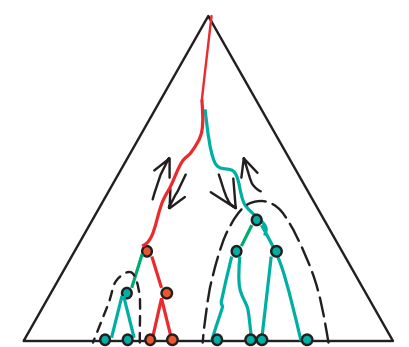

(b) Refitting a tree in post-order

Figure 3. Updating BVH. (a) The nodes in red are leaves whose primitives are to be deformed, inserted, or removed due to mesh refinement. (b) A subtree in red is labeled. The nodes in green are in inactive status; the nodes in red are in active status. The labeled subtree is traversed in post-order to refit the BVH. The subtree in red is for collision query. 
operation is that when no primitive bounded by a node or by its descendants is in the mesh at the current resolution, the bounding box of the node cannot be used in collision query. Therefore, the node is set to inactive. When a primitive bounded by a node or by its descendants is to be inserted into or deformed in the mesh at the current resolution, the bounding box of the node needs to be refitted before the collision query is performed. Therefore, the node is set to deformed.

3. ACTIVE-REFIT $(x)$ calculates the smallest AABB that encloses range $[$ left $[x]]$ and range $[$ right $[x]]$ and sets status $[x]$ to active.

4. ACTIVE-WALK $(x)$ post-order traverses the subtree $T_{s}$ from the root node. For a node $x$ in $T_{s}$, if status $[x]$ is deformed, $\operatorname{ACTIVE-REFIT~}(x)$ is called. This operation returns a refitted tree $T$ as shown in Figure 3(b). The status of any nodes in $T$ is either active or inactive. Collision query is applied to only those nodes whose status is active. Therefore, when the resolution of the mesh becomes coarser, the number of nodes whose status fields are active is decreased. The running time cost of the collision detection is reduced.

5. ACTIVE-UPDATE $(I, R, D, T)$ calls $\operatorname{ACTIVE-MARK}(I, R, D, T)$ to mark the AB-Tree $T$. Then it calls ACTIVE-WALK $(x)$ to refit the subtree $T_{s}$ of $T$. The purpose is to refit the BVH.

6. ACTIVE-INTERSECT $\left(T, T^{\prime}\right)$ performs an intersection test between two AB-Trees $T$ and $T^{\prime}$. For each visited pair of nodes, if the status field of each node is active, the two bounding boxes are tested for overlap. Only the nodes for which the bounding boxes overlap are further traversed. If both nodes are leaves and their primitives intersect, the indices of the two leaves are returned.

\subsubsection{Complexity Analysis}

The new operation we need to analyze is $\operatorname{ACTIVE-UPDATE}(I, R, D, T)$. The pseudocode is given in Algorithms 1-3.

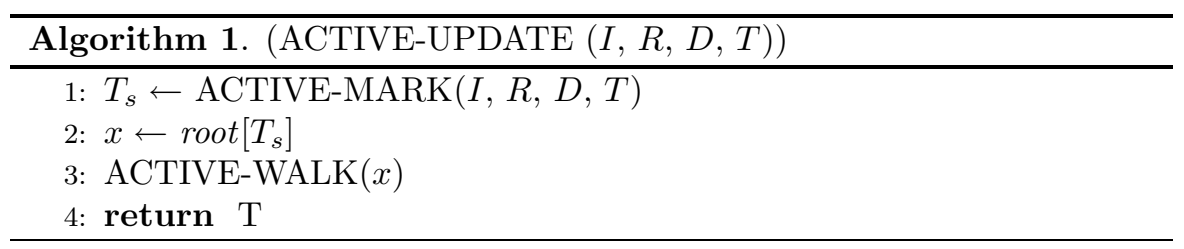



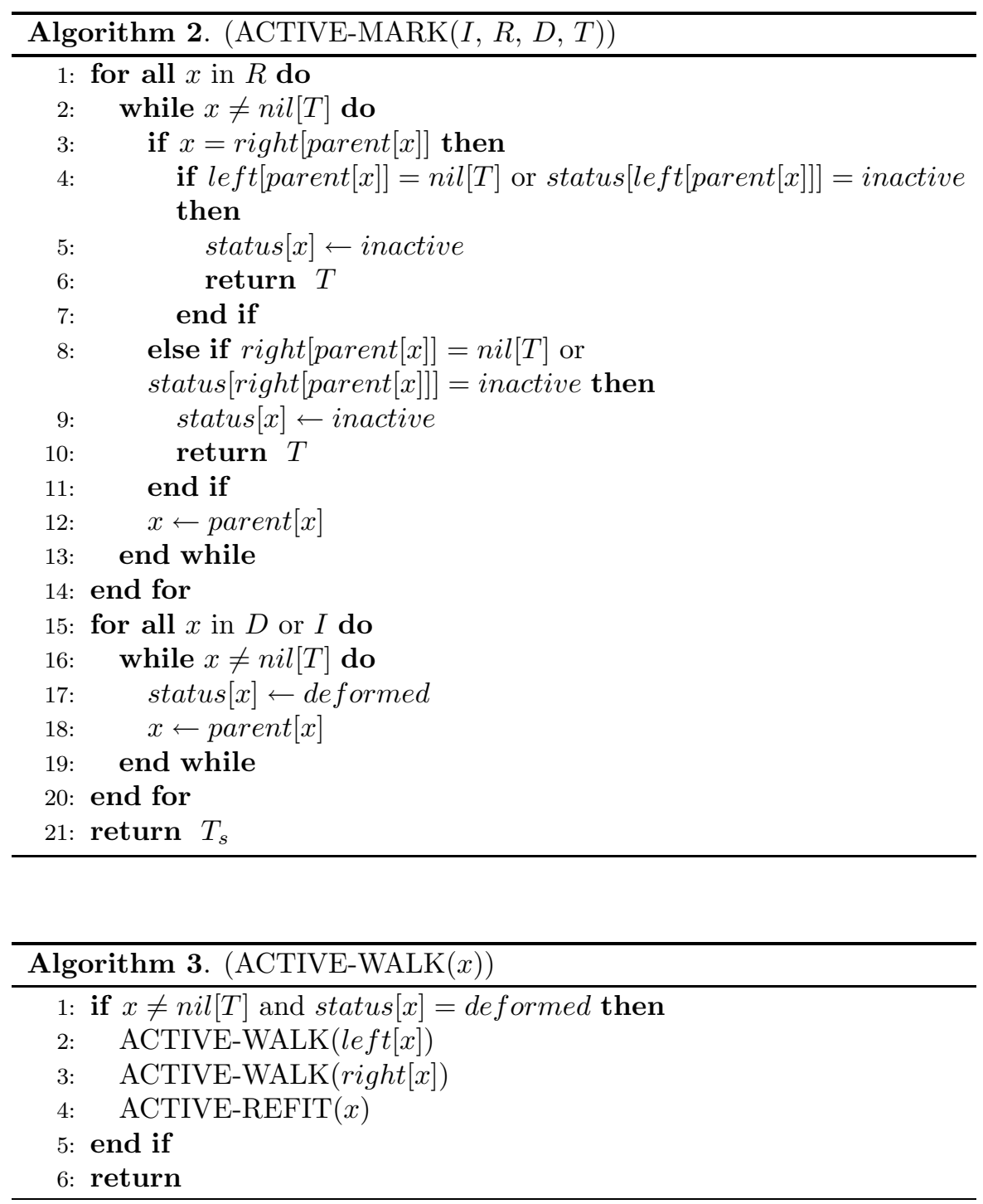

The complexity of operation ACTIVE-REFIT $(x)$ is not affected by the total number of nodes in $T$. Hence, we consider the cost of this operation as a constant. The following theorem gives a proof that $\operatorname{ACTIVE-UPDATE}(I, R$, $D, T)$ takes $O(k(\log n-\log k+2))$ time in the worst case to refit an AB-Tree.

Theorem 1. Let $T$ be an AB-Tree. $L$ is the set of all leaves in the tree $T$ whose primitives are in a mesh that tree $T$ is built upon. If $I, R$, and $D$ are 
three subsets of $L, R \cap D=\varnothing, R \cap I=\varnothing$, and $I \cap D=\varnothing$, then the operation ACTIVE-UPDATE $(I, R, D, T)$ takes $O(k(\log n-\log k+2))$ time in the worst case, where $n$ is the total number of leaves in $T, k$ is the number of primitives to be deformed in, removed from, or inserted into the mesh. Furthermore, if $k$ is set to be in a range $[1, K]$, where $K$ is a constant and $K<<n$, then the call takes $O(\log n)$ time.

Proof. We consider the maximum number of calls to ACTIVE-REFIT $(x)$ as the worst-case running time of the algorithm. The main idea of the proof is to find out the maximum number of nodes whose field status is deformed.

Let $T_{1}$ be the running time of $\operatorname{ACTIVE-UPDATE}(I, R, D, T)$. Let $k_{1}$ be the size of $R, k_{2}$ be the size of $I$, and $k_{3}$ be the size of $D$, then $k=k_{1}+k_{2}+k_{3}$. The total number of for loops in $\operatorname{ACTIVE-MARK}(I, R, D, T)$ is $k$. In the second for loop of lines 15-20, when $k_{1}=0$, the maximum number of loops is $k$. Since an AB-Tree $T$ is an AVL tree and $n$ is the number of leaves in $T, T$ has height $O(\log n)$. For the first loop, the maximum number of assignments in line 17 is $\left(\log _{2} n+1\right)$. For the second loop, the maximum number of assignments in line 17 is $\left(\log _{2} n+1\right)-2^{0}$. For the third and the fourth loops, the maximum number of assignments in line 17 is $\left(\log _{2} n+1\right)-2^{1}$. By induction, when $k=2^{b}$, where $b$ is a nonnegative integer, the maximum number of operations in line 17, measured as the running time of $\operatorname{ACTIVE-UPDATE}(I, R, D, T)$, is

$$
\begin{aligned}
T_{1}(n, k) & =k\left(\log _{2} n+1\right)-\sum_{i=0}^{\log _{2} k} 2^{i-1} i \\
& =k\left(\log _{2} n-\log _{2} k+2\right)-1 \\
& =O(k \log n-k \log k+2 k) .
\end{aligned}
$$

If $k$ is set to be in a range [1,K], where $K$ is a constant and $K<<n$, then the call to ACTIVE-UPDATE $(I, R, D, T)$ takes $O(\log n)$ time.

Using an AB-Tree, time-critical collision detection can be performed on models in multiresolution representation. In Section 4, we introduce the adaptive LOD selection method for multiresolution collision detection.

\section{Adaptive LOD Selection}

LOD selection (or LOD management) for real-time rendering of large-scale virtual environment and related perceptual issues have received a lot of attention since the mid-1970s. LOD management deals with assigning LODs to objects based on a series of criteria. At runtime, visually less important objects are assigned low LODs and vice versa. Funkhouser and Séquin propose 
some criteria, such as semantics, focus, motion, and hysteresis, to calculate the visual importance of objects [Funkhouser and Séquin 93]. Reddy uses distance and projected screen space to manage LODs [Reddy 01]. Brown et al. introduced an attention model for LOD selection [Brown et al. 03]. Gaze direction is another criterion to LOD management [Luebke et al. 00]. Researchers have also begun to study the issue of perceptually adaptive techniques for realistic collision simulation in scenes with large numbers of animated objects. O'Sullivan and Dingliana investigated different factors that affect collision perception, including eccentricity, separation, distracters, causality, and accuracy of collision response [O'Sulivan and Dingliana 01]. The accuracy of a computed collision response is directly dependent on the LOD to which a collision detection algorithm applies. A lower LOD results in more perceivable inconsistency in physics simulation. For example, one of the possible consequences of reducing the accuracy of $\mathrm{CD}$ is that objects bounce off each other at a distance, leaving a perceivable gap. Therefore, maintaining the level of collision detection detail as close to the LOD for rendering as possible can generate a visually more realistic simulation. Based on their observation, we present a LOD selection method for collision detection. The purpose is to accelerate collision detection and maintain a physically plausible animation system.

\subsection{Selection Criteria}

By studying the different factors that affect the believability of simulation, we can assign a measure of importance to different objects in a scene. More important objects are allocated more LODs in high priority, thus more accurate $\mathrm{CD}$ is achieved. The accuracy of CD for less important objects is sacrificed for saving processing time of $\mathrm{CD}$ for the entire scene, thus a target frame rate is obtained. In our implementation, the measure of importance is affected by three factors.

1. Viewing distance: Based on human vision perceptional capability, collisions happening on near objects attract more attention than those happening on far objects. Therefore, we give an object low priority as it moves away from the viewpoint and give high priority to the object as it moves toward the viewpoint.

2. Application context: The idea is to classify each object by its context in an application. Collisions will attract more attention when they happen on important objects. Therefore, more important objects are assigned higher priority. For example, in a video game, collisions of human avatars with other objects attract the most attention from the viewer, therefore the objects have the highest priority, other moving ob- 
jects have the second highest priority, and static objects and background scene, such as wall and ceiling, have the lowest priority.

3. Motion: Objects that are moving quickly across the screen appear blurred and can be seen for only a short amount of time. A viewer may not be able to see them clearly. In implementation, we determine the effect of blur on the viewer by simply measuring the length of motion path of objects in screen space of previous frames. Higher priority is assigned to less blurred objects.

\subsection{LOD Selection Model}

We present a LOD selection model based on the above mentioned observation in the form of heuristic, aiming at finding a combination of continuous LODs and the AB-Tree algorithm for all objects in a scene that produces the most realistic collision simulation within a target animation frame. The model is formulated as following.

In a virtual environment, the frame time $T$ for collision detection can be expressed as

$$
T=T_{R}+T_{B}+T_{Q}+T_{L O D}
$$

where $T_{R}$ is the time for mesh refinement, $T_{B}$ is the time for BVH refitting, $T_{Q}$ is the time for collision queries, and $T_{L O D}$ is the time for LOD selection. $T_{R}$ is proportional to the number of vertex split (or edge collapse) operations needed to refine a mesh from one LOD to another. When LODs do not change drastically between neighboring frames, $T_{R}$ is roughly a small constant and $T_{B}$ is in the order of logarithm of the size of the full meshes. $T_{Q}$ is determined by not only mesh resolution but also collision configurations. $T$ may be set fixed or variable depending on the stability of the costs of other tasks in an animation frame. The LOD for each object has to be selected during $T_{L O D}$. The LOD selection model calculates LODs in linear time to the number of objects in the scene.

An object is defined as $O(\alpha, \beta, \gamma, L O D 1, L O D 2)$, where $\alpha$ is its current viewing distance, $\beta$ is its application context, $\gamma$ is the length of the twodimensional projection of its motion path in previous frames, $L O D 1$ is the LOD of the previous frame, and LOD2 is the LOD of the current frame. In preprocessing, each object is classified by its application context into three categories: most important (Q1), where $\beta=1$, less important (Q2), where $\beta=2$, and not important (Q3), where $\beta=3$. In each frame, the measured time for $\mathrm{CD} T^{\prime}$ in the previous frame is compared with $T$. The result falls into one of the following three cases. 
1. $T^{\prime}-T \leq-t$

2. $T^{\prime}-T>0$

3. $T^{\prime}-T \leq 0$ and $T^{\prime}-T>-t$, where $t$ is a threshold parameter

In Case 1, the LODs for the current frame are to be increased exponentially. In Case 2, the LODs are to be reduced exponentially. In Case 3, the LODs remain unchanged. We deal with the first two cases differently. Let $(0, D)$ be visible range, $W$ be the diagonal length of screen space, and $\triangle L O D$ be the number of vertex split or edge collapse operations. In our implementation, $\triangle L O D$ equals the total number of the operations of a model divided by 50 , and $t$ equals $10 \%$ of $T$. A smaller $\triangle L O D$ means visually the accuracy of CD is changed more smoothly. In Case 1,

$$
L O D 2=L O D 1+\Delta L O D \times f(\alpha, \gamma) \times g(i, j, \beta)
$$

In Case 2, the time cost for CD needs to be reduced quickly in order to guarantee an interactive rate. Therefore, we simplify the calculation of LODs and accelerate the reduction of LODs in comparison with Case 1:

$$
\begin{aligned}
L O D 2 & =L O D 1+\Delta L O D \times g(i, j, \beta), \\
f(\alpha, \gamma) & =\min \left(\frac{D-\alpha}{D}, \frac{W-\gamma}{W}\right), \\
g(i, j, \beta) & =\eta_{1} \times g_{1}(i, j, \beta)+\eta_{2} \times g_{2}(i, j),
\end{aligned}
$$

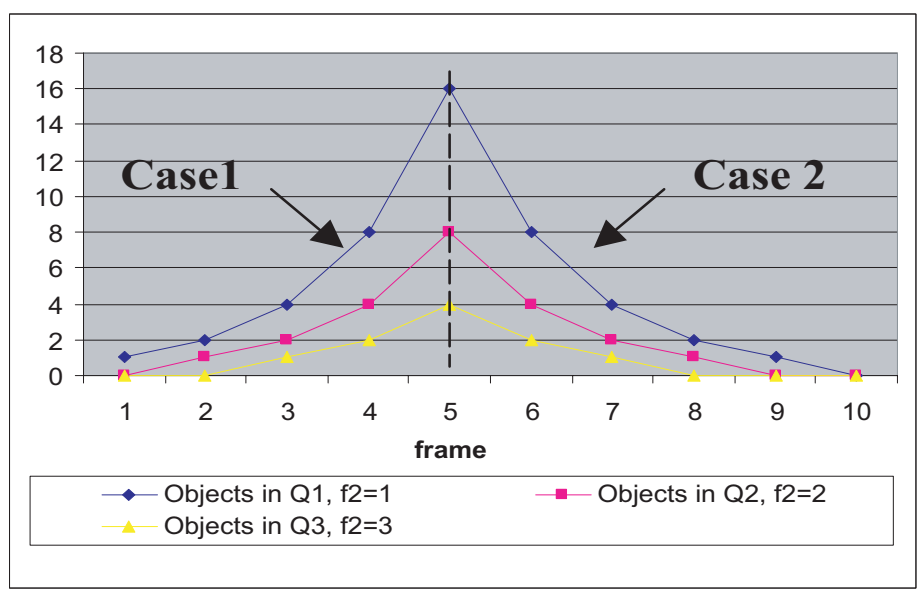

Figure 4. Illustration of function $g(i, j, \beta)$. 
where $\eta_{1}+\eta_{2}=1$. In Case $1, \eta_{1}=1$ and $\eta_{2}=0$. In Case $2, \eta_{1}=0$ and $\eta_{2}=1$.

$$
\begin{aligned}
g_{1}(i, j, \beta) & =\left\{\begin{array}{l}
2^{i-j-\beta}, i-j \geq \beta, i=1,2,3, \ldots, n, \text { and } j=1,2,3, \ldots, n \\
2^{0}, i-j<\beta, i=1,2,3, \ldots, n, \text { and } j=1,2,3, \ldots, n
\end{array}\right. \\
g_{2}(i, j) & =\left\{\begin{array}{l}
2^{2 j-i-1}, 2 j \geq i+1, i=1,2,3, \ldots, n, \text { and } j=1,2,3, \ldots, n \\
2^{0}, 2 j<i+1, i=1,2,3, \ldots, n, \text { and } j=1,2,3, \ldots, n
\end{array}\right.
\end{aligned}
$$

where $i$ is the index of the current frame, $n$ is the total number of frames, and $j$ is the index of the closest previous frame of $i$ with a condition that in frame $j+1$, the case is changed from 1 or 3 to 2 , or from 2 or 3 to 1 . The function $g(i, j, \beta)$ is illustrated in Figure 4, in which $j$ is 5 .

In order to maintain the time cost of collision queries in a frame to be above a threshold, the resolution for each model has to be changed dynamically. In system initialization phase, each model is refined to the coarsest resolution. Then if computational resources are available, the models are refined to higher resolution by following the LOD selection method introduced in this section. In such a way, the gap between the LOD for rendering and the LOD for collision detection is reduced.

\section{Implementation and Results}

In this section, we describe the implementation of $\mathrm{AB}$-Tree and demonstrate our simulation results.

\subsection{System Demonstration}

We have applied our collision detection approach to PM on a variety of models listed in Table 1. We have performed three demonstrations with the listed models on a Pentium4 $2.8 \mathrm{GHz}$ processor PC with $510 \mathrm{MB}$ memory, an NVIDIA GeForce FX5200 graphics card, and Windows XP OS. Our implementation uses $\mathrm{C}++$ and the OpenGL library for physics simulation and graphics rendering. Interference detection using BVH has been studied exten-

\begin{tabular}{|l|l|l|l|}
\hline Models & Bones & Cow & Bunny \\
\hline \# faces in $M_{n}$ (original mesh $)$ & 4204 & 5804 & 57563 \\
\hline \# vertices in $M_{n}$ (original mesh $)$ & 2154 & 2904 & 25000 \\
\hline \# faces in $M_{0}$ (base mesh) & 10 & 10 & 500 \\
\hline \# vertices in $M_{0}$ (base mesh $)$ & 39 & 8 & 1406 \\
\hline
\end{tabular}

Table 1. Parameter settings for models. 


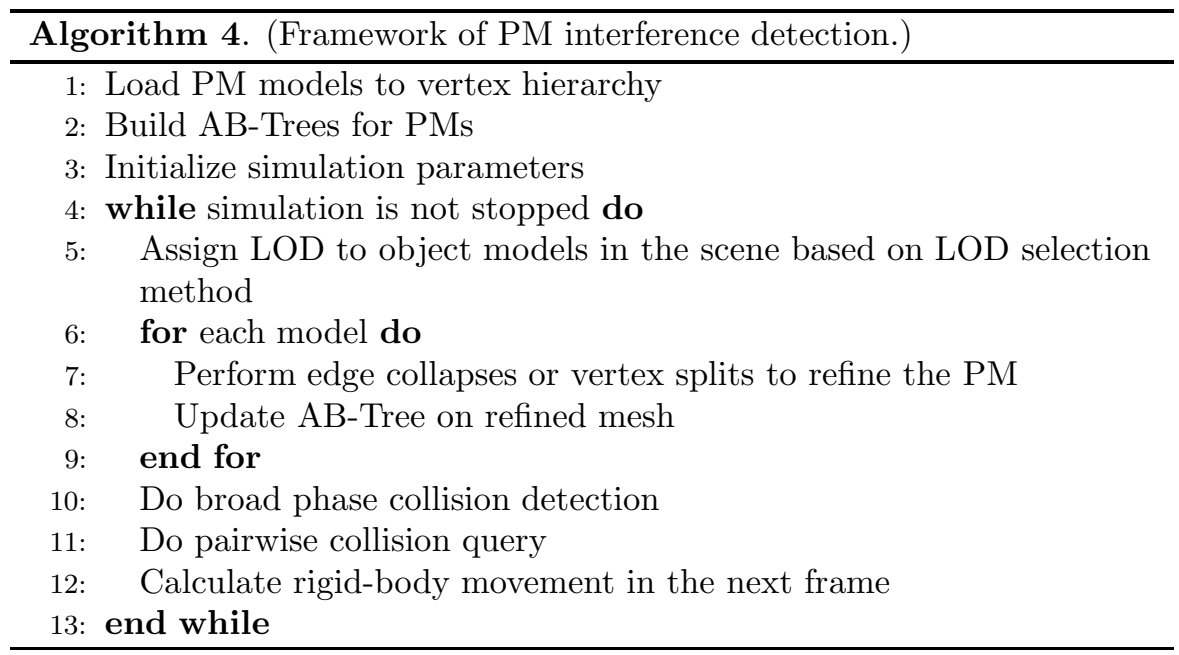

sively in the literature. In this paper, we focus on analyzing the complexity of refitting a BVH at runtime and measuring the CPU time cost of this task. A framework of PM interference detection is given in Algorithm 4.

\subsection{Runtime Performance}

We have successfully applied our approach to PM interference detection on the benchmarks given in Section 5.1. A benchmark model is shown in Figure 5. The performance of a demonstration is determined by the complexity of the geometric models and the granularity of the continuously refined LODs.

We have performed a measurement in CPU time of the collision detection task on both the static LOD representations and the PM representations at the same resolution. We do not observe a performance loss of the PM models, which use a complex data structure in comparison with a simple data structure used by the static LOD. On the other hand, when the resolution of a PM decreases, the performance of the PM interference detection is improved significantly. When a PM gets coarser, the number of pairwise BV interference detections and the number of pairwise primitive interference detections are decreased. Therefore, the running time is decreased.

We have also performed a complexity analysis on the dynamic BVH refitting algorithm described in Section 3.2 and compared the theoretical result with experimental results. Figure 6 shows a comparison between the running time cost given in Equation (1) and the CPU time of the BVH refitting for each model listed in Section 5.1 when the PMs are refined at different granularity. 

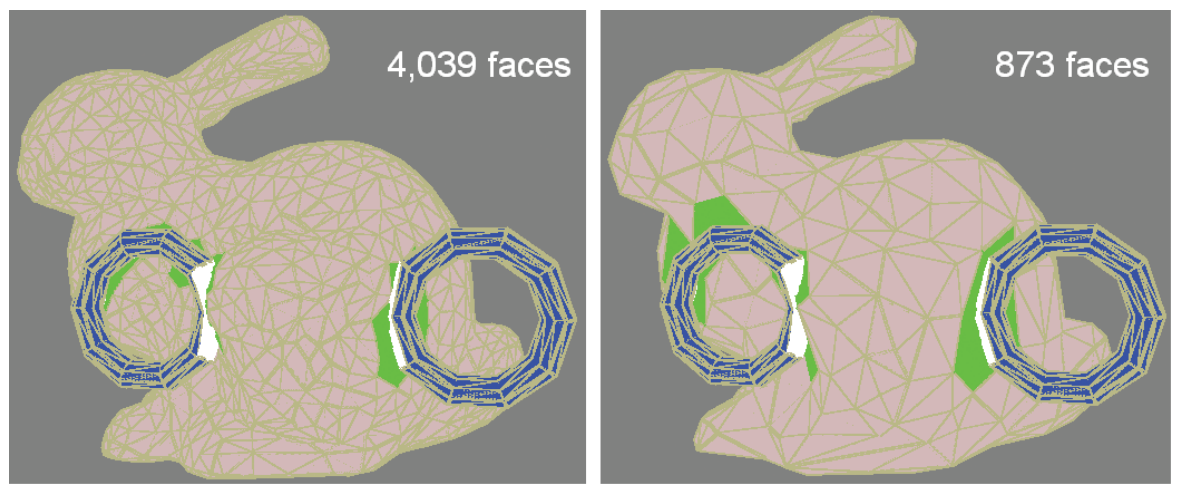

Figure 5. Multiresolution collision detection. Left: Moving tori in contact with bunny at high resolution. Right: BVH is refitted when the model is refined to a low resolution. The interference triangle faces (in green and white) are changed accordingly when the LOD is switched.

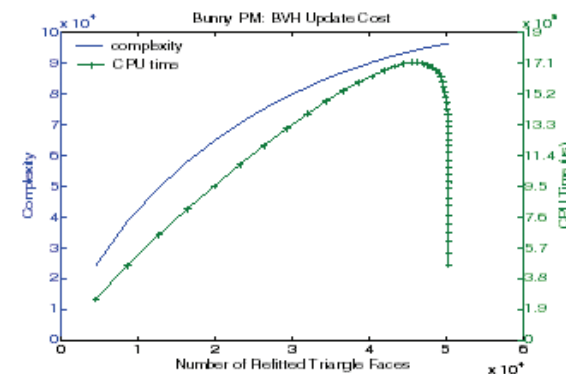

(a) Bunny mode1

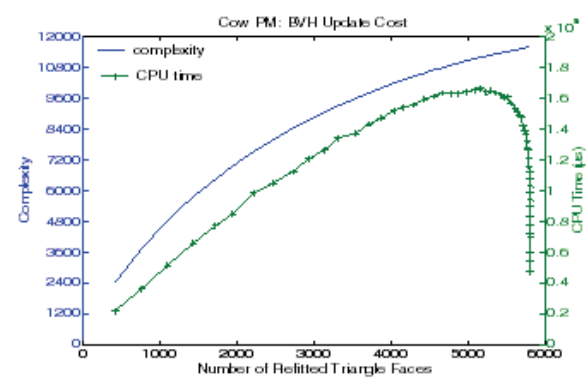

(b) Cow model

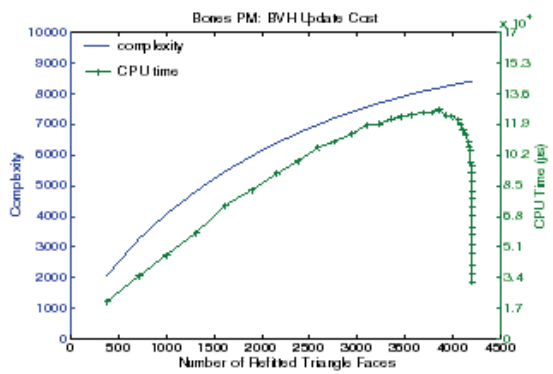

(c) Bones model

Figure 6. Comparison of CPU time and the complexity of the BVH refitting. 
It is not difficult to see that the CPU time is increased nearly proportional to the analytical results when $k$, the number of leaves to be marked in the $\mathrm{BVH}$, increases except for the cases when $k$ is very close to $n$. Typically $k$ is set to be less than a constant which is much smaller than $n$. Therefore we believe that strong agreements are achieved. Furthermore, we observe that when $k$ is smaller than $10 \%$ of the total number of faces in the original mesh, the updating operation requires less than $15 \%$ query time. In practical computer simulation and animation applications such as video games, the context of the models does not change very often and the movement configuration of the models does not change swiftly. The granularity of the continuously refined LODs is not too large relative to the number of faces in a mesh, which means $k$ is relatively small. Therefore, our collision detection approach can perform fast and robustly in these applications.

In comparison to existing time-critical approaches for static LODs, our new approach has the following advantages.

1. Physically more accurate simulations are generated because collision response calculation is on instantaneous impulse mesh models rather than BVs of the mesh primitives.

2. The computational cost is substantially reduced by partially refitting BVH with a worst-case complexity in the order of logarithm when the LODs of objects do not change drastically.

3. Since the BVHs are dynamically refitted according to the adaptively refined LOD, perceptual inconsistency is removed successfully. In addition, our approach can handle nonmanifold triangle meshes in arbitrary topology.

Acknowledgments. We are grateful to the LORNET NSERC Research Network for supporting this research.

\section{References}

[Bradshaw and O'Sullivan 04] G. Bradshaw and C. O'Sullivan. "Adaptive MedialAxis Approximation for Sphere-Tree Construction." ACM Trans. on Graphics $23: 1$ (2004), 1-26.

[Brown et al. 03] R. Brown, L. Cooper, and B. Pham. "Visual Attention-Based Polygon Level of Detail Management." In Proc. of the 1st International Conference on Computer Graphics and Interactive Techniques in Australasia and South East Asia, pp. 55-62. New York: ACM Press, 2003. 
[El-Sana and Varshney 00] J. El-Sana and A. Varshney. "Continuously-Adaptive Haptic Rendering." In Virtual Environments, pp. 135-144, Aire-la-Ville, Switzerland: Eurographics Association, 2000.

[Funkhouser and Séquin 93] T. A. Funkhouser and C. H. Séquin. "Adaptive Display Algorithm for Interactive Frame Rates During Visualization of Complex Virtual Environments." In Proceedings of SIGGRAPH 93, Computer Graphics Proceedings, Annual Conference Series, edited by James T. Kajiya, pp. 247253, New York: ACM Press, 1993.

[Garland 04] Michael Garland. "Quadric Error Metrics." Available online (http:// graphics.cs.uiuc.edu/ garland/research/quadrics.html), 2004.

[Garland and Heckbert 97] M. Garland and Paul S. Heckbert. "Surface Simplification using Quadric Error Metrics." In Proceedings of SIGGRAPH 97, Computer Graphics Proceedings, Annual Conference Series, edited by Turner Whitted, pp. 209-216, Reading, MA: Addison Wesley, 1997.

[Gottschalk et al. 96] S. Gottschalk, M. Lin, and D. Manocha. "OBB-Tree: A Hierarchical Structure for Rapid Interference Detection." In Proceedings of SIGGRAPH 96, Computer Graphics Proceedings, Annual Conference Series, edited by Holly Rushmeier, pp. 171-180, Reading, MA: Addison Wesley, 1996.

[Gottschalk et al. 97] S. Gottschalk, M. C. Lin, and D. Manocha. "RAPID: Robust and Accurate Polygon Interference Detection." Available online (http://www. cs.unc.edu/ geom/OBB/OBBT.html), 1997.

[Gross et al. 96] M. Gross, O. Staadt, and R. Gatti. "Efficient Triangular Surface Approximations using Wavelets and Quadtree Structures." IEEE Trans. on Visual and Computer Graphics 2:2 (1996), 130-144.

[Hecker 00] C. Hecker. "Physics in Computer Games." Communications of the ACM 43:7 (2000), 34-39.

[Hoppe 96] H. Hoppe. "Progressive Meshes." In Proceedings of SIGGRAPH 96, Computer Graphics Proceedings, Annual Conference Series, edited by Holly Rushmeier, pp. 99-108, Reading, MA: Addision Wesley, 1996.

[Hoppe 97] H. Hoppe. "View-Dependent Refinement of Progressive Meshes." In Proceedings of SIGGRAPH 97, Computer Graphics Proceedings, Annual Conference Series, edited by Turner Whitted, pp. 189-198, Reading, MA: Addision Wesley, 1997.

[Hubbard 96] P. M. Hubbard. "Approximating Polyhedra with Spheres for TimeCritical Collision Detection." ACM Trans. on Graphics 15:3 (1996), 179-210.

[Jiménez et al. 01] P. Jiménez, F. Thomas, and C. Torras. "Collision Detection: A Survey." Computers and Graphics 25:2 (2001), 269-285.

[Klosowski et al. 98] J. T. Klosowski, M. Held, J. Mitchell, H. Sowizral, and K. Zikan. "Efficient Collision Detection Using Bounding Volume Hierarchies of k-DOPs." IEEE Trans. on Visualization and Computer Graphics 4:1 (1998), $21-36$. 
[Larsen et al. 99] E. Larsen, S. Gottschalk, M. Lin, and D. Manocha. "Fast Proximity Queries with Swept Sphere Volumes." Tech. Rep. TR99-018, Dept. of Comput. Sci., Univ. North Carolina, 1999.

[Lin and Gottschalk 98] M. Lin and S. Gottschalk. "Collision Detection between Geometric Models: A Survey." In Proc. of IMA Conference on Mathematics of Surfaces, pp. 37-56. [CITY]: [PUB], 1998.

[Luebke and Erikson 97] D. Luebke and C. Erikson. "View-Dependent Simplification of Arbitrary Polygonal Environments." In Proceedings of SIGGRAPH 97, Computer Graphics Proceedings, Annual Conference Series, edited by Turner Whitted, pp. 199-208. Reading, MA: Addision Wesley, 1997.

[Luebke et al. 00] D. Luebke, B. Hallen, D. Newfield, and B. Watson. "Perceptually Driven Simplification Using Gaze-Directed Rendering." Tech. Rep. CS-2000-4, University of Virginia, 2000.

[Luebke et al. 02] D. Luebke, M. Reddy, J. Cohen, A. Varshney, B. Watson, and R. Huebner. Level of Detail for 3D Graphics. San Francisco: Morgan Kaufmann, 2002.

[O'Sulivan and Dingliana 01] C. O'Sulivan and J.Dingliana. "Collisions and Perception." ACM Trans. on Graphics 20:3 (2001), 151-168.

[Otaduy and Lin 03] M. A. Otaduy and M. C. Lin. "CLODs: Dual Hierarchies for Multiresolution Collision Detection." In Proc. of Eurographics Symposium on Geometry Processing, pp. 94-101. Aire-la-Ville, Switzerland: Eurographics Association, 2003.

[Reddy 01] M. Reddy. "Perceptually Optimized 3D Graphics." IEEE Computer Graphics and Applications 21:5 (2001), 68-75.

[Taubin and Rossignac 98] G. Taubin, J. Rossignac. "Geometric Compression Through Topological Surgery." ACM Trans. on Graphics 17:2 (1998), 84-115.

[van den Bergen 97] G. van den Bergen. "Efficient Collision Detection of Complex Deformable Models using AABB Trees." journal of graphics tools 2:4 (1997), $1-13$.

[van den Bergen 02] G. van den Bergen. "Software Library for Interference Detection." Available online (http://solid.sourceforge.net/), 2002.

[Xia et al. 97] J. Xia, J. El-Sana, and A. Varshney. "Adaptive Real-Time Level-ofDetail-Based Rendering for Polygonal Models." IEEE Trans. on Visualization and Computer Graphics 3:2 (1997), 171-183.

\section{Web Information:}

Pseudocode and screenshots of the presented algorithm are available at http://jgt. akpeters.com/papers/LiuEtAl06/.

Peiran Liu, University of Ottawa, [ADDRESS] 
(peiran@discover.uottawa.ca)

Nicolas D. Georganas, University of Ottawa, [ADDRESS]

(georganas@discover.uottawa.ca)

Gerhard Roth, National Research Council of Canada, [ADDRESS]

(Gerhard.Roth@nrc-cnrc.gc.ca)

Received July 21, 2004; accepted in revised form November 1, 2005. 